normalized for head size, as percentiles with respect to an agematched healthy population. Results: Accuracy and reproducibility results are presented in Tables 1-2. Figure 1 illustrates a test-retest example. Sensitivity between controls and AD patients was high: all measurements (cross-sectional and longitudinal) showed significant differences between the two groups (p-value $<1 \mathrm{e}-5$ ); see figure 2. Effect sizes are given in Table 3 and ROC curves in Figure 3. Conclusions:icobrain's substructure volumetry demonstrates high accuracy and reproducibility, while also ensuring significant sensitivity between $\mathrm{AD}$ and controls. The icobrain volume signature may assist detecting deviations from normal brain aging. References: [1] Lysandropoulos AP, Absil J, Metens T, et al. Brain and Behavior. 2016;6(2):e00422. https://doi.org/10.1002/brb3.422. [2] Smeets D, Ribbens A, Sima DM, et al. Brain and Behavior. 2016;6(9):e00518. https://doi.org/10.1002/brb3.518. [3] Sima D., Jain S., Maertens A., Van Vlierberghe E., Van Hecke W., Smeets D. ECTRIMS 2016; 145739.

\section{P3-410 COGNITIVE RESERVE, BRAIN RESERVE AND BRAIN MAINTENANCE: MOVING FROM CONCEPT TO MEASUREMENT}

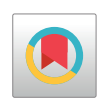

Evan Fletcher ${ }^{1}$, Laura B. Zahodne ${ }^{2}$, Timothy J. Hohman ${ }^{3}$, Brandon E. Gavett ${ }^{4}$, Charlie S. DeCarli ${ }^{1}$, Dan Mungas ${ }^{5},{ }^{1}$ University of California, Davis, Davis, CA, USA ${ }^{2}$ University of Michigan, Ann Arbor, MI, USA; ${ }^{3}$ Vanderbilt University, Nashville, TN, USA;

${ }^{4}$ University of Colorado Colorado Springs, Colorado Springs, CO, USA; ${ }^{5}$ University of California, Davis, Sacramento, CA, USA.

Contact e-mail: evanfletcher@gmail.com

Background: Cognitive reserve, brain reserve and brain maintenance are related concepts that have been used to account for inter-individual differences in cognition faced with disease and aging. Brain reserve refers to brain resources available to support cognition in the face of aging and disease. Brain maintenance focuses on preservation of brain integrity: decreased susceptibility to changes from pathology or aging. A recent operational definition of cognitive reserve is residual variance in episodic memory performance not explained by a priori brain structure and demographic variables. Here, we use voxel-based regression analyses to identify cross-sectional and longitudinal brain structure variables that should be included in operational measurements of reserve. Methods: In a database of 320 older participants, we residualized baseline memory performance and memory rate of longitudinal change (i.e. slope) for baseline cortical gray matter (GM) volume, racial/ethnic group, gender, and education. Memory change was additionally residualized for longitudinal atrophy rate of global cortical GM. Next, we studied correlations of voxel-based cortical GM with the baseline memory residual, and voxel-based longitudi-

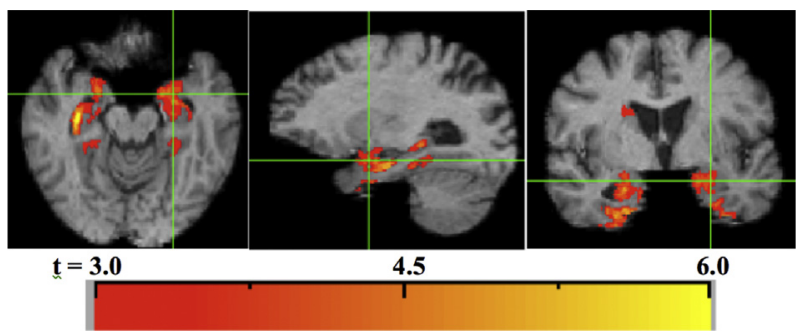

Figure 1. Brain reserve associations. Significant clusters of voxel-based associations between GM thickness and baseline memory residuals. Color bar shows levels of $\mathrm{t}$-thresholds for clusters.

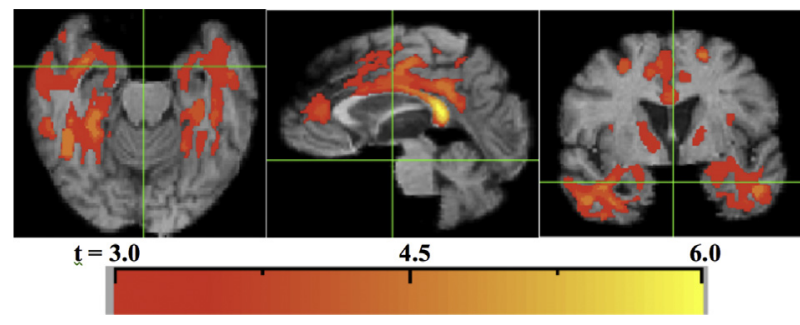

Figure 2. Brain maintenance associations. Significant clusters of voxelbased associations between longitudinal atrophy rates and memory decline residuals. Color bar shows levels of t-thresholds for clusters.

nal atrophy rates with the memory change residual. Results: Voxelbased analyses revealed specific brain regions that were significantly correlated with residualized baseline memory (Fig. 1) and residualized memory decline (Fig. 2). Thus, residual variation in baseline memory was further explained by GM thickness of medial temporal lobe (MTL) regions, including entorhinal cortex, hippocampus and parahippocampal gyri. Residual variation in memory change was further explained by atrophy in extensive temporal lobe regions, the splenium and posterior corpus callosum, the cingulate cortex and components of the default mode network (DMN), including medial prefrontal cortex and posterior cingulate cortex. Conclusions: Our results suggest that GM thickness in MTL regions is an important marker of brain reserve, above and beyond global GM volume (Fig. 1). Low atrophy rates of temporal regions, as well as in the splenium, cingulum bundle and portions of the DMN (Fig. 2), are important markers of brain maintenance, above and beyond global GM atrophy. Voxel-based analyses that better capture regional components of brain reserve and brain maintenance can incrementally improve cross-sectional and longitudinal operationalization of cognitive reserve and maintenance.

\section{P3-411 CLINICAL SIGNIFICANCE OF IN-VIVO STAGING OF REGIONAL AMYLOID DEPOSITION IN SUBJECTIVE MEMORY COMPLAINERS}

Fatemah A. Sakr ${ }^{1}$, Michel J. Grothe ${ }^{2}$, Enrica Cavedo ${ }^{3,4,5,6}$, MarieOdile Habert ${ }^{7,8,9}$, Hugo Bertin ${ }^{9}$, Maxime Locatelli ${ }^{7,8,9}$, Stephane Lehéricy ${ }^{10,11,12}$, Bruno Dubois ${ }^{3,5,6}$, Stefan J. Teipel ${ }^{2,13}$, Harald Hampel ${ }^{3,4,5,6},{ }^{1}$ Rostock University, Rostock, Germany; ${ }^{2}$ German Center for Neurodegenerative Diseases, Rostock, Germany; ${ }^{3}$ Sorbonne Université, Assistance Publique - Hôpitaux de Paris, Alzheimer Precision Medicine, Hôpital de la Pitié-Salpêtrière, Paris, France; ${ }^{4}$ AXA Research Fund and Sorbonne Université Chair, Paris, France, ${ }^{5}$ Institut du Cerveau et de la Moelle Épinière, INSERM, CNRS, Paris, France; ${ }^{6}$ Institut de la Mémoire et de la Maladie d'Alzheimer, Département de Neurologie, Hôpital de la Pitié-Salpêtrière, AP-HP, Paris, France $;{ }^{7}$ Assistance Publique Hôpitaux de Paris, Hôpital Pitié-Salpêtrière, Département de Médecine Nucléaire, Paris, France; ${ }^{8}$ Sorbonne Universités, University Pierre and Marie Curie, Centre National de la Recherche Scientifique, INSERM, Laboratoire d'Imagerie Biomédicale, Paris, France; ${ }^{9}$ Centre pour l'Acquisition et le Traitement des Images, Paris, France; ${ }^{10}$ CENIR Neuroimaging Research Center, Hôpital de la Salpêtriere, Paris, France; ${ }^{11}$ INSERM, Institut du Cerveau et de la Moelle Epiniere, Paris, France; ${ }^{12}$ Sorbonne Universites, Universite Pierre et Marie Curie, Paris, France; ${ }^{13}$ Department of Psychosomatic Medicine, Rostock University Medical Center, Rostock, Germany.Contacte-mail: fatemah.sakr@med. uni-rostock.de 